\title{
Cutaneous and giant hepatic haemangioma associated with Kasabach-Merritt syndrome in an adult patient with discoid lupus erythematosus
}

\author{
Wei Gao', Xihu Qin² \\ 'Department of Dermatology, The Affiliated Changzhou No. 2 People's Hospital of Nanjing Medical University, Changzhou, Jiangsu, \\ China \\ 2Derpartment of General Surgery, The Affiliated Changzhou No. 2 People's Hospital of Nanjing Medical University, Changzhou, Jiangsu,
} China

Adv Dermatol Allergol 2021; XXXVIII (5): 909-911

DOI: https://doi.org/10.5114/ada.2021.110150

A 57-year-old female, presented with a dark redness mass with adherent scale and unclear boundaries on the right cheek for 11 years. The patient had complained of swelling and pain of the mass for 2 months. Because of the low platelet count $\left(40-60 \times 10^{9} / 1\right.$; norm: $100-300 \times$ $10^{9} /$ l), the patient could not have the skin biopsy of the lesion of the right cheek and was transferred to the inpatient department of haematology for medical treatment. Her past history revealed no other illnesses except that she had bruised easily and suffered from petechiae and ecchymoses of the body frequently for 29 years. The patient was diagnosed as thrombocytopenia by bone marrow biopsy. After being treated with oral steroid therapy, although the platelet count did not return to normal completely, the condition turned better.

Clinical examination showed that the cutaneous lesion appeared as a dark purplish-red mass with swelling, slight concavity in the middle with a small amount of scale, unclear boundaries, and a firm texture, located on the right cheek (Figure 1 A). The physical examination showed no hepatosplenomegaly.

On laboratory examination, the platelets were $40 \times$ 10\% / (norm: 100-300 × 10\%/l), haemoglobin $85 \mathrm{~g} / \mathrm{l}$ (norm: 110-150 g/l), and white blood cells (WBC) $3.0 \times 10^{9} / \mathrm{l}$ (norm: 4-10 × 109/l). Coagulation profile showed fibrinogen $3.9 \mathrm{~g} / \mathrm{l}$ (norm: 2.0-4.0 g/l), D-dimer 9.74 mg/l (norm: 0-0.55 mg/l), prothrombin time (PT) $12 \mathrm{~s}$ (norm: 9.0-13 s), activated partial thromboplastin time (APTT) $21.9 \mathrm{~s}$ (norm: 19.0-34.5 s). Serum immunological tests was negative. Bone marrow biopsy showed hyperplastic anaemia, thrombocytopenia (megakaryocyte increased). B-mode ultrasonography of the cutaneous lesion showed an abnormal structure of subcutaneous tissue, foliar margins hypoechoic area in the centre, unclear boundaries, with irregular shape, no obvious capsule and $2.7 \mathrm{~cm} \times 1.0 \mathrm{~cm}$ core size, which was considered as right cheek haemangioma (Figure 1 B). Computed tomography $(\mathrm{CT})$ scan revealed multiple hepatic haemangiomas of different sizes, one of them invading the left lobe of the liver, $14 \mathrm{~cm}$ in diameter (Figure 1 C).

The patient was initially diagnosed as adult KasabachMerritt syndrome (KMS) associated with cutaneous and giant hepatic haemangiomas. The patient was treated with low dose i.v. heparin (500 IU/h), high dose methylprednisolone (2 mg/kg/day) and received single donor platelets 1 unit and red blood cell 5 unit transfusions. This treatment was maintenance treatment for nearly 3 weeks, resulting in a slight increase of fibrinogen levels and improvement of the platelet count. Afterwards, the left hepatic lobe tumour and spleen of the patient were removed by excision surgery. Histopathologic examination of the liver tumour after operation confirmed the hepatic cavernous haemangioma. Microscopically, there were dilated blood vessel cavities, extensive thrombosis and organization, normal spleen in size and shape. One month after the operation, the routine blood examination returned to normal gradually. We also found that after the resection of the liver tumour, the swelling mass of the right face had disappeared, while the dark redness macula with scale still existed (Figure $1 \mathrm{D}$ ). The pathology of the skin biopsy of the macula on the right face showed mild hyperplasia of epidermis, follicular keratotic plugging, liquefaction degeneration of basal cells, incontinence of pigment and focal lymphocytic infiltration around appendage (Figure $1 \mathrm{E}$ ). Direct immunofluorescence (DIF) test showed granular IgG, IgM, C3 deposition, the basement membrane zone broadening. On the basis of clinical characteristics, labora-

Address for correspondence: Xihu Qin, Department of General Surgery, The Affiliated Changzhou No. 2 People’s Hospital of Nanjing Medical University, Xing Long Road 29, Changzhou, Jiangsu, 213003, China, e-mail: qinxihu11@163.com Received: 21.09.2019, accepted: 3.03.2020. 

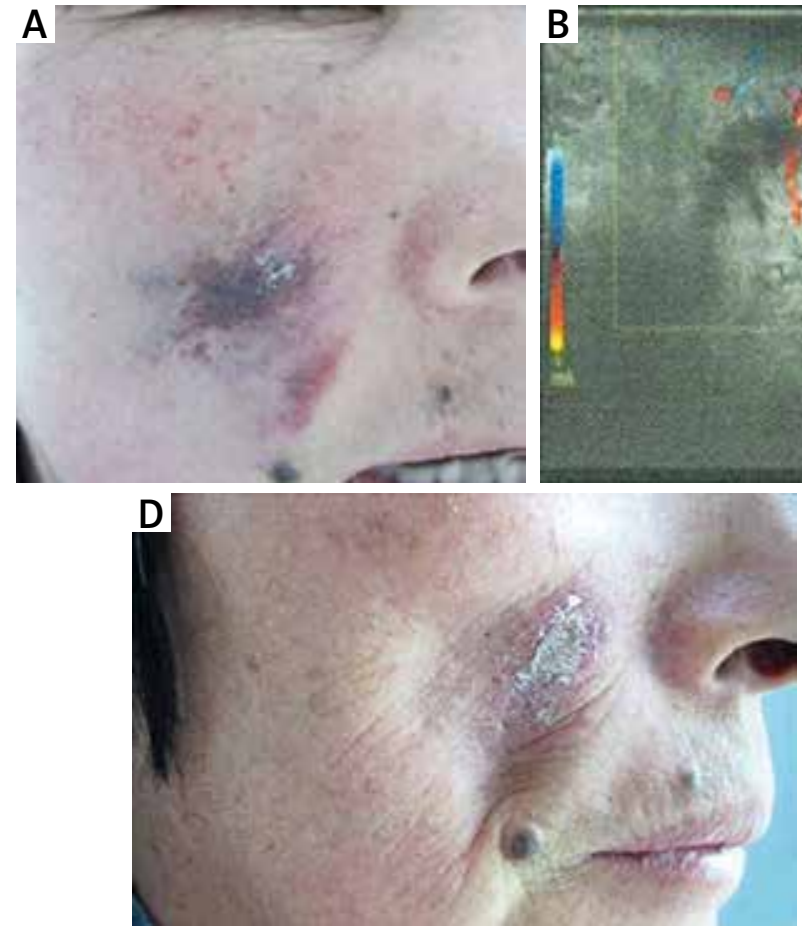
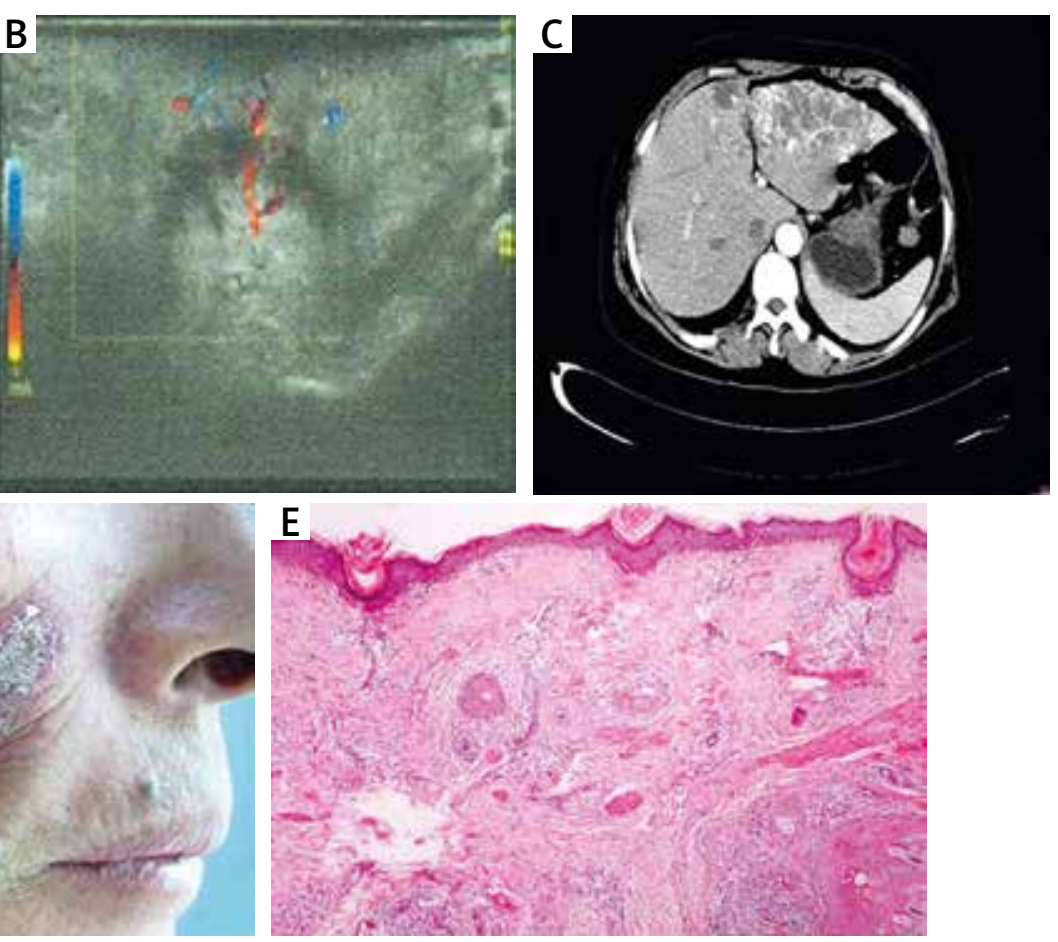

Figure 1. Cutaneous lesion. A - A dark purplish-red mass with swelling, slight concavity in the middle with a small amount of scale, unclear boundaries, and a firm texture on the right cheek. B - B-mode ultrasonography of the cutaneous lesion. The lesion was $2.7 \mathrm{~cm} \times 1.0 \mathrm{~cm}$ in core size and showed expansion of the central hypoechoic area in all the directions, unclear boundaries, irregular shape, no obvious capsulation, and high levels of blood flow signs. C - CT scan of the liver and spleen. There was a large hepatic haemangioma invading the left lobe of the liver, $14 \mathrm{~cm}$ in diameter. D - The mass of the right cheek disappeared with centre atrophy depression and one dark erythema with some scale on the right cheek without obvious atrophy depression. $\mathbf{E}$ - The pathology of the macula on the right face. It showed mild hyperplasia of epidermis, follicular keratotic plugging, liquefaction degeneration of basal cells, incontinence of pigment and focal lymphocytic infiltration around appendage

tory examination, pathological and DIF manifestations, the patient was diagnosed as discoid lupus erythematosus (DLE) with adult KMS associated with cutaneous and giant hepatic haemangioma. Thereafter, the patient was given tacrolimus ointment for 2 months and was recommended to stay away from sunlight, the skin lesion faded away.

The patient suffered from cutaneous and giant hepatic haemangiomas which were associated with KMS, the combination of vascular tumours and thrombocytopenic coagulopathy [1] and complicated with DLE on the right cheek at the same position as the cutaneous haemangiomas. To date, although many cases of KMS have been described, mostly in infants with cutaneous haemangiomas, hepatic haemangiomas are seldom associated with KMS and diffuse intravascular coagulopathy. Totally, there were 11 papers about adult KMS associated with giant hepatic haemangioma over the past 20 years [2]. This syndrome may result in a lifethreatening multi-organ haemorrhage and overall mortality (estimated to be 30\%) [3]. This patient accepted oral steroid treatment discontinuously, prolonging the duration of the disease.
The aetiology of KMS is unknown and the pathogenic mechanism of the syndrome relates to proliferation of the abnormal vascular endothelium cells within the haemangioma. Different from the normal hepatic cavernous haemangioma, the pathological manifestations of the patient showed wide intralesional thrombosis and organization, suggesting continued consumption of platelets and clotting factors and fibrinolysis, according with high D-dimer level, which may lead to intralesional haemorrhage and enlargement of the vascular tumour [4]. Although we cannot distinguish which kind of haemangioma occurred first, we suppose that she had suffered from thrombocytopenia frequently for 29 years also in association with hepatic haemangiomas, bringing consumption of platelets, and then leading to the cutaneous haemangioma, which disappeared after giant hepatic haemangioma operation.

Currently, there are no consensus guidelines for the management of KMS. Some studies have reported comprehensive therapy, including steroid therapy, interferon, arterial embolization, vincristine, radiotherapy, and surgery [5-7]. Hepatic cavernous haemangiomas are the 
most usual type of benign hepatic tumour, presenting with KMS infrequently encountered, so their treatment is challenging and controversial, especial those giant ones. Surgery is the most appropriate method for our case, because of the tumour location.

After the successful treatment of the KMS, although the platelet count became normal, the facial haemangioma disappeared miraculously, the facial dark redness macule with adherent scale still often occurred, especially when exposed to the sunlight for a long time. After taking the skin biopsy and DIF test with normal autoantibodies at the Institute of Dermatology, the patient was diagnosed as DLE. Tracing the history of the patient and initial expression of the cutaneous lesion, the adherent scale needed to be noticed, which indicated that DLE had happened for many years. One study showed that SLE is associated with multiple hepatic haemangiomas and suggested that SLE may be directly implicated in the formation of benign vascular neoplasia, which may be related to increased circulating levels of oestrogens and other angiogenic factors [8]. So whether the DLE is related to the haemangioma, or it is only a coincidence, the patient still needs to be followed up for a long time.

Because of the complex condition of the patient, it takes a long time to establish the absolutely right diagnosis and provide the correct treatment. KMS is a complicated disease owing to its mortality and difficult management modalities. Surgical treatment is still controversial but the only curative modality. Luckily, our patient recovered well after the resection of the haemangioma and spleen. So our case highlights that early and careful recognition of the cutaneous hallmarks, offered an opportunity for early and correct diagnosis of the lifethreatening disease.

\section{Acknowledgments}

This study was supported by Changzhou Sci\&Tech Program (Grant No. CJ20210163).

\section{Conflict of interest}

The authors declare no conflict of interest.

\section{References}

1. Enjolras O, Mulliken JB, Wassef M. Residual lesions after Kasabach-Merritt phenomenon in 41 patients. J Am Acad Dermatol 2000; 42: 225-35.

2. Liu X, Yang Z, Tan H, et al. Giant liver hemangioma with adult Kasabach-Merritt syndrome: case report and literature review. Medicine 2017; 96: e7688.

3. Oak CY, Jun $\mathrm{CH}$, Cho EA, et al. Hepatic hemangioma with Kasabach-Merritt syndrome in an adult patient. Korean J Gastroenterol 2016; 67: 220-3.

4. Hall GW. Kasabach-Merritt syndrome: pathogenesis and management. Br J Haematol 2001; 112: 851-62.
5. Yoon HS, Lee JH, Moon HN, et al. Successful treatment of retroperitoneal infantile hemangioendothelioma with Kasabach-Merritt syndrome using steroid, $\alpha$-interferon, and vincristine. J Pediatr Hematol Oncol 2009; 49: 852-54.

6. Hauer J, Graubner U, Konstantopoulos N, et al. Effective treatment of kaposiform hemangioendotheliomas associated with Kasabach-Merritt phenomenon using four-drug regimen. Pediatr Blood Cancer 2007; 49: 852-4.

7. Yang Z, Tan H, Liu X, et al. Extremely giant liver hemangioma $(50 \mathrm{~cm})$ with Kasabach-Merritt syndrome. J Gastrointest Surg 2017; 21: 1748-9.

8. Berzigotti A, M Frigato, E Mnfredini, et al. Liver hemangioma and vascular liver diseases in patients with systemic lupus erythematosus. Word I Gastroenterol 2011; 17: 4503-8. 